\title{
Erratum: Topological data analysis of contagion maps for examining spreading processes on networks
}

Dane Taylor, Florian Klimm, Heather A. Harrington, Miroslav Kramár, Konstantin Mischaikow, Mason A. Porter \& Peter J. Mucha

Nature Communications 6:7723 doi: 10.1038/ncomms8723 (2015); Published 21 Jul 2015; Updated 4 Sep 2015

In the sixth paragraph of the Methods section of this article, there is a typographical error in the equation $[1-q(t) / N] d^{\mathrm{NG}}$. The correct version of the equation reads as follows:

$$
[1-q(t) / N]^{d^{(\mathrm{NG})}}
$$

